\title{
Die Rückschreibung zur Rekonstruktion der Gewannflurgenese im bernischen Seeland
}

\author{
(Kartenbeilage)
}

\section{Fragestellung}

Den außerordentlichen Landschafts- und Siedlungsveränderungen der letzten Jahrzehnte wird vielfach noch eine hohe Stabilität der älteren Siedlungs- und Flurformen bis ins 19., teilweise bis ins 20. Jahrhundert gegenübergestellt.

Am Beispiel des bernischen Seelandes, insbesondere an den Fluren von Gampelen, Gals und Ins, soll auf die folgenden Fragen eingegangen werden:

1. Wie dynamisch verlief die mittelalterlich-frühneuzeitliche Flurentwicklung in einem traditionellen Gewannflurgebiet?

2. Gibt es Hinweise auf den Ursprung des Gewannflursystems?

3. Veränderte sich die Siedlungstextur, d. h. lassen sich Ausbausiedlungen oder Ortswüstungen nachweisen?

Im Gegensatz zu zahlreichen Planfluren, deren Ursprung vielfach in schriftlichen Quellen, später sogar in Karten und Plänen festgehalten ist, liegt der Ursprung und die Genese der Gewannflursysteme weitgehend im dunkeln. Dies, obschon die Gewannfluren zu den häufigsten Flurformen in Mitteleuropa und auch im schweizerischen Mittelland gehören. BRÜHWILER vermutete, daß ihr Ursprung wahrscheinlich überhaupt nicht werde geklärt werden können (BRÜHWILER 1975: 35).

Unter Gewannflursystem verstehen wir nicht nur die schmalstreifige Besitzparzellierung, in unserem Untersuchungsgebiet seit dem 16. Jahrhundert meist als Blockgewanne ausgebildet, sondern auch der genossenschaftlich geregelte dreijährige Fruchtwechsel in den Ackerarealen (Flurzwang) und die gemeinsame Weide in der Brachzelg und auf der Allmende'.

Wo die einzelnen Besitzparzellen noch wesentlich größer, meist noch blockförmig sind, und jeder Landwirtschaftsbetrieb nur eine oder wenige Parzellen besaß in den einzelnen Zelgen, sprechen wir von Dreizelgensystem ${ }^{2}$. Und schließlich bezeichnen wir die Wirtschaftsform des Einzelhofes mit arrondierter Flur, dessen Ackerareal im dreijährigen Zyklus angebaut wird, als Dreifelderwirtschaft ${ }^{3}$.

\section{Methode}

In der historisch-genetischen Kulturlandschaftsforschung sind Ergebnisse meist nur durch die Kombination ganz unterschiedlicher Methoden zu erzielen, da einerseits die kulturräumlichen und andererseits die naturräumlichen Faktoren untersucht werden müs$\operatorname{sen}^{4}$.

Je nach spezifischer Fragestellung, besonders aber aufgrund der Quellenlage, steht jedoch in der Regel eine Methode im Vordergrund.

In Abbildung 1 wird schematisch gezeigt, welche Methoden in dieser Untersuchung eingesetzt wurden und zu Ergebnissen führten.

Da für die gesamte ehemalige Herrschaft Erlach die Bodenzinsbücher vom 16. bis ins 18. Jahrhundert fast lückenlos vorhanden sind und zudem das Katasterplanwerk von 1779/1786 im Maßstab ca. 1:1200 mit der letzten Urbaraufnahme korrespondiert, stand die sogenannte «Rückschreibung» ${ }^{5}$ für das 16 . bis 18. Jahrhundert im Zentrum. In Abbildung 2 ist diese Methode schematisch dargestellt, zudem sind die wichtigsten Quellen und die analytischen Ergebnisse für das 16. Jahrhundert eingetragen.

Die Beschreibung der einzelnen Besitzparzellen seit 1533/1566 (je nach Grundherrschaft) und die jeweilige Bezugnahme der neuen Urbare auf ihre Vorgänger ermöglichten neben der Rekonstruktion der Parzellarpläne auch die parzellenweise Kartierung der Landnutzung.

Und weil in den Urbaren einerseits bei jeder Parzelle der Besitzer angegeben ist, und andererseits die Parzellen seit dem 16. Jahrhundert unverändert $\mathrm{zu}$ Bodenzinseinheiten zusammengefaßt und meist mit der älteren Gutsbezeichnung versehen $\operatorname{sind}^{6}$, konnten auch die frühen Eigentums- und Besitzverhältnisse ${ }^{7}$ parzellenweise analysiert werden. Für die Zeit vor 1530 wurden hauptsächlich die schriftlichen Quellen

Hans-Rudolf Egli, Dr., Geographisches Institut der Universität Bern, Hallerstraße 12, CH-3012 Bern 


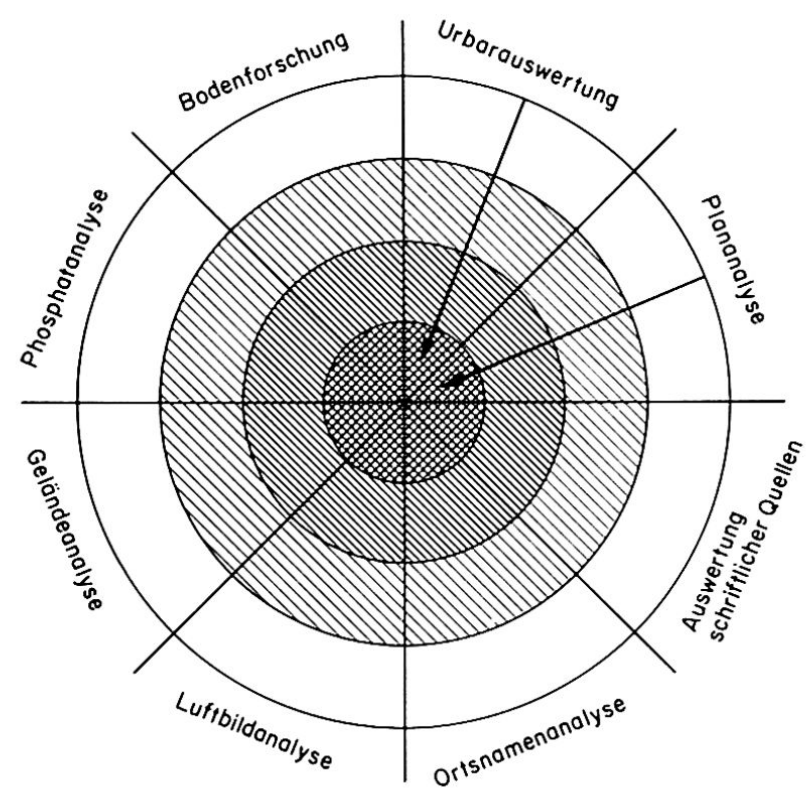

16. - 18. Jahrhundert

gesicherte Aussage

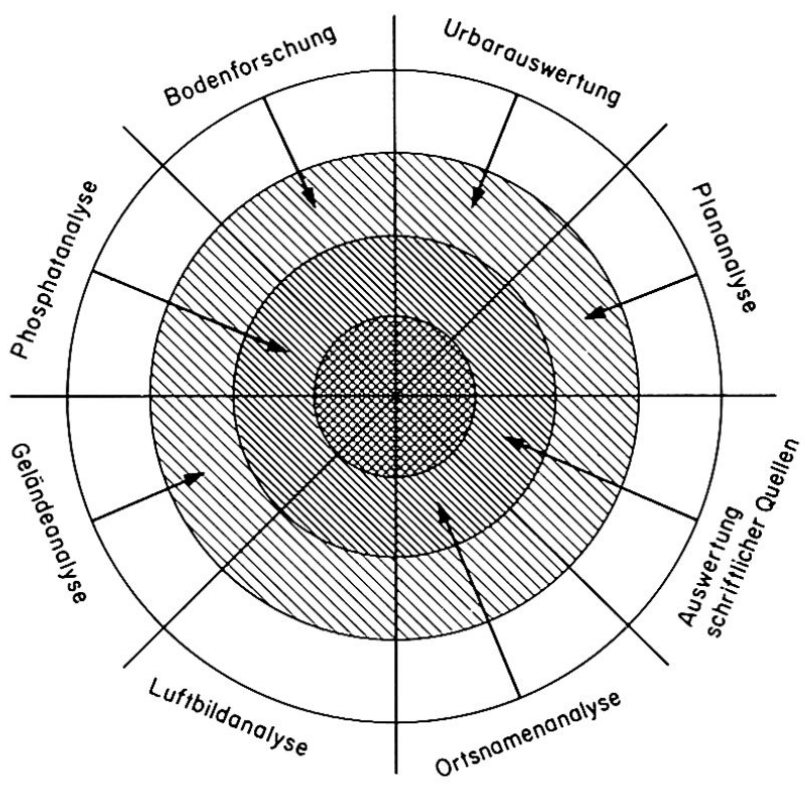

12. - 15. Jahrhunder

MIV vermutete Aussage mögliche Aussage

Abb. 1 Schematische Darstellung der Methodenkombination zur Untersuchung der Gewannflurgenese im bernischen Seeland in zwei verschiedenen Perioden.

untersucht und die Flurnamen analysiert. Zudem wurden die anthropogenen Reliktformen im Gelände kartiert und die naturräumliche Eignung für die mittelalterliche Landwirtschaft untersucht. Die Phosphatanalyse von Bodenproben ${ }^{8}$ und einzelne Profilschnitte sollten Hinweise auf Ortswüstungen bestätigen.

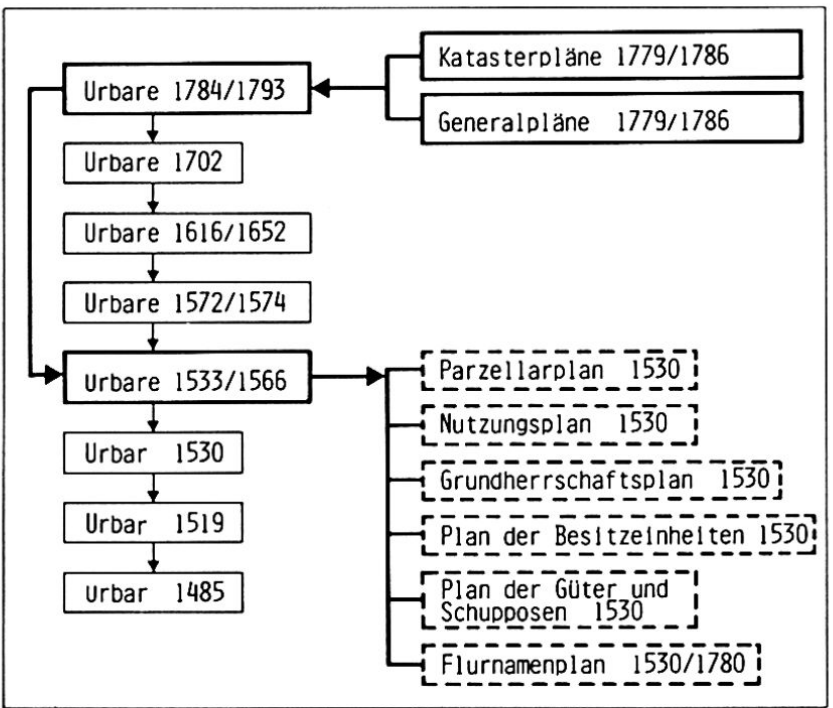

Abb. 2 Schematische Darstellung der Rückschreibung vom 18. ins 16. Jahrhundert aufgrund der Quellen der alten Herrschaft Erlach.

\section{Ergebnisse}

Die drei benachbarten, auf der Beilagenkarte dargestellten Fluren von Gals, Gampelen und Ins waren im 18. Jahrhundert ganz unterschiedlich groß. Die 151,8 ha von Gals waren in 1009 Besitzparzellen aufgeteilt, das 133,0 ha große Flurareal von Gampelen umfaßte 853 Parzellen und zur außerordentlich großen Flur von Ins (834,5 ha) gehörten 5595 Parzellen. Da nur ganz unbedeutende Veränderungen der Flurareale zwischen 1530 und 1780 festgestellt wurden, konnte aufgrund der Teilungsverhältnisse der bodenzinspflichtigen Parzellen auch die Parzellenzahl angenähert für 1530 berechnet werden: In Gals waren es rund 480 Parzellen $(1: 2,11)$, in Gampelen 310 $(1: 2,76)$ und in Ins etwa $2690(1: 2,08)$. Die Besitzparzellen waren trotz Realerbteilung im Verlaufe von 250 Jahren durchschnittlich nur ein- bis zweimal geteilt worden. Das heißt, daß in allen Fluren bereits im 16. Jahrhundert eine ausgeprägte Kurzgewannflur vorlag, und daß auch bei denjenigen Erbgängen mit mehr als einem Nachkommen die einzelnen Parzellen meistens nicht aufgeteilt, sondern den einzelnen Erben zugeteilt wurden. Die Erbleihe, bei der die Betriebe innerhalb der Familie weitervererbt wurden, war nicht durch eine kurzfristigere Leiheform abgelöst worden. Auch die Flurnutzung erwies sich für das 16. bis 18. Jahrhundert als außerordentlich stabil (EGLI 1983: 67f.). In Tabelle 1 sind die Nutzungsanteile und Zelgengrößen zusammengefaßt dargestellt. 
Tab.1 Die Größe der Nutzungsareale in Hektaren der Fluren von Gals, Gampelen und Ins vom 16. bis 18. Jahrhundert

\begin{tabular}{|c|c|c|c|c|c|c|c|}
\hline & \multicolumn{3}{|l|}{ Acker } & \multirow[t]{2}{*}{ Wiesen } & \multirow[t]{2}{*}{ Reben } & \multirow{2}{*}{$\begin{array}{l}\text { Beunden } \\
\text { Gärten }\end{array}$} & \multirow{2}{*}{$\begin{array}{l}\text { Flurfläche } \\
\text { total }\end{array}$} \\
\hline & 1. Zelg & 2. Zelg & 3. Zelg & & & & \\
\hline $\begin{array}{l}\text { Gals } \\
\text { Gampelen } \\
\text { Ins }\end{array}$ & $\begin{array}{r}31,3 \\
12,5 \\
131,9\end{array}$ & $\begin{array}{r}34,3 \\
26,4 \\
206,2\end{array}$ & $\begin{array}{r}24,3 \\
19,6 \\
173,2\end{array}$ & $\begin{array}{r}48,1 \\
48,2 \\
223,0\end{array}$ & $\begin{array}{r}1,3 \\
19,5 \\
73,1\end{array}$ & $\begin{array}{r}12,5 \\
6,8 \\
27,1\end{array}$ & $\begin{array}{l}151,8 \\
133,0 \\
834,5\end{array}$ \\
\hline
\end{tabular}

Die relativ großen Flächenunterschiede der drei Zelgen in allen Fluren zeigen, $\mathrm{da} ß$ die theoretisch gleich großen Schläge der Dreifelder- bzw. Dreizelgenwirtschaft in Wirklichkeit oft nicht existierten ${ }^{9}$. Die durch die unterschiedlichen Anbauflächen bedingten Ertragsunterschiede konnten wahrscheinlich über die Saatgutmenge teilweise ausgeglichen werden, da die Nutzungsintensität nicht mit heutigen Verhältnissen verglichen werden kann. Zudem waren die klimatisch bedingten Ertragsunterschiede noch größer als diejenigen der Anbaufläche ${ }^{10}$. Wie aus der Karte ersichtlich ist, gehörten in Gampelen zwei Ackerareale zur ersten Zelg und drei Areale zur zweiten Zelg. In Ins war das «Brühlzelgli» südlich des Dorfes im gleichen Anbauzyklus wie das "Erlachfeld». Aus der Tabelle 1 und aus der Karte geht auch die unterschiedliche Bedeutung des Rebbaus hervor. In Gals, das am nördlichen Hangfuß des Jolimonts liegt, spielte der Weinbau praktisch keine Rolle, wogegen in Ins das Rebareal rund einer halben und in Gampelen einer ganzen Ackerzelg entsprach.

Besonders aufschlußreich für die Gewannflurgenese ist die Rekonstruktion der Besitzverhältnisse. In der Karte haben wir drei unterschiedliche Entwicklungsstände dargestellt: für Gampelen die Besitzstreuung ausgewählter Betriebe für das Jahr 1784, für Gals vier ehemalige "Güter» aus dem 16. Jahrhundert und für Ins die Zinseinheiten des «Hofgutes» im Zustand von 1530, das zumindest im Kernbestand ins 12. Jahrhundert zurückreicht.
Aus der Tabelle 2 ist die außerordentliche Besitzzersplitterung in Gampelen von 1530 bis 1784 ersichtlich. Drei der vier dargestellten Landwirtschaftsbetriebe (Hans Jacob Käch, Johann Wilhelm Wenker, Christian Johann Gyger) besaßen je von allen Gütern des 16. Jahrhunderts Parzellen. Die meisten Güter wurden als «Schupposen» bezeichnet und trugen den Namen des zinsverantwortlichen Trägers bei der Urbaraufnahme von 1530. Bei diesen Schupposen, die teilweise synonym als «Gut» bezeichnet sind, handelt es sich nicht um alte Landwirtschaftsbetriebe, da wir für die ganze Herrschaft Erlach in den Quellen des 14. und 15. Jahrhunderts den Begriff «Schuppose» kein einziges Mal fanden und wir keine der im 12. Jahrhundert genannten Schupposen mit einer späteren Wirtschaftseinheit identifizieren konnten (EGLI 1983: 115).

Alle vier Betriebe streuen ziemlich regelmäßig über die gesamte Flur: im Ackerland, im Wiesland und in den Reben. Sämtliche Parzellen sind schmale Streifen und weniger als $200 \mathrm{~m}$ lang. Dies dürfte auch mit der engen Lage zwischen dem Steilhang des Jolimonts und dem Flachmoor des Großen Mooses zusammenhängen.

In der Flur von Gals sind die vier ersten Wirtschaftseinheiten der Tabelle 3 kartographisch dargestellt. Diese machen rund $60 \%$ der Parzellen der gesamten Flur aus und zeigen die Streuung der Landwirtschaftsbetriebe bei der ersten parzellenweisen Urbaraufnahme um 1530. Alle Güter liegen in ausgeprägter Gemengelage und streuen ebenfalls über die gesamte

Tab. 2 Die Güterzersplitterung in Gampelen im Jahre 1784 am Beispiel von vier ausgewählten Betrieben

\begin{tabular}{|c|c|c|c|c|}
\hline \multirow{2}{*}{$\begin{array}{l}\text { Herkunft der Parzellen } \\
\text { aufgrund der Gutsbezeichnung } \\
\text { um } 1530\end{array}$} & \multicolumn{4}{|c|}{ Besitzer und Anzahl Parzellen im Jahre 1784} \\
\hline & H.J.Käch & J.W. Wenker & Chr. J. Gyger & W. Dietrich \\
\hline von «Rubelis Schuppose» & 17 & 2 & 1 & 5 \\
\hline von "Grebers Gut»" & 7 & 1 & 2 & 2 \\
\hline von «Dietrichs Schuppose" & 4 & 3 & 2 & - \\
\hline von «Mügelis Schuppose» & 15 & 12 & 6 & 1 \\
\hline von “Gygers Schuppose» & 9 & 5 & 2 & - \\
\hline von "Mosers Schuppose" & 9 & 12 & 7 & 2 \\
\hline von "Meisters Schuppose» & 9 & 5 & 5 & 1 \\
\hline von «Bendicht Kalis Schuppose» & 13 & 8 & 5 & - \\
\hline von «Wenkers Schuppose» & 3 & 5 & 4 & 2 \\
\hline von "Hans Kalis Schuppose" & 10 & 2 & 2 & 1 \\
\hline von “Taubis Schuppose” & 8 & 2 & 2 & 2 \\
\hline von “Stürlers Schuppose» & 9 & 3 & 4 & - \\
\hline von weitern Gütern & 9 & 13 & 4 & - \\
\hline Total & 122 & 73 & 46 & 16 \\
\hline
\end{tabular}

Quelle: Erlach-Urbare Nr. 29a und 112 
Tab. 3 Die Zersplitterung und Bodenzinsbelastung der "Güter" und "Schupposen" von Gals um 1530

\begin{tabular}{|c|c|c|c|c|c|c|c|c|c|}
\hline & \multirow{3}{*}{$\begin{array}{l}\text { Anzahl } \\
\text { Parzellen }\end{array}$} & \multicolumn{8}{|c|}{ Lehenszins } \\
\hline & & \multicolumn{4}{|l|}{ in $M a ̈ \beta^{a)}$} & \multirow[b]{2}{*}{ Hühner } & \multirow[b]{2}{*}{ Eier } & \multirow[b]{2}{*}{ Schilling } & \multirow[b]{2}{*}{ Pfennige } \\
\hline & & Weizen & Korn & Hafer & Nüsse & & & & \\
\hline "Schreyers Gut» & 48 & 70 & & & & $21 / 2$ & 50 & 100 & \\
\hline "Tribolet Gut" & 134 & 72 & 93 & 24 & 6 & $61 / 2$ & 130 & 145 & \\
\hline "Tschasselets Güter» & 98 & & 72 & & & $31 / 2$ & 70 & 168 & \\
\hline "WyBbrots Gut" & 54 & 13 & & & & 1 & 20 & 113 & 8 \\
\hline «Schreyers Schuppose» & 8 & 7 & & & & $3 \frac{1}{2}$ & 70 & 1 & \\
\hline "Kremers Gut" & 20 & 23 & & & & & & 19 & 10 \\
\hline "Großmeisters Gut" & 58 & & 29 & & & 2 & 40 & 98 & 6 \\
\hline "Trinina Gut»" & 16 & 18 & & & & & & 20 & \\
\hline "Meisters Gut" & 18 & & 28 & & & 1 & 20 & 3 & \\
\hline "Anna Wileneta Gut» & 18 & 12 & & & & & & 24 & \\
\hline "Glado Martins Schuppose" & 18 & 16 & & & & $1 / 2$ & 10 & 46 & 10 \\
\hline "Mogellis Gut" & 19 & 12 & & & & 1 & 20 & 24 & \\
\hline "Gerharts Gut» & 42 & 31 & & & & $31 / 2$ & 70 & 79 & \\
\hline 8 kleine Zinseinheiten & 13 & 3 & & & & 1 & 20 & 40 & 1 \\
\hline Total & 564 & 277 & 222 & 24 & 6 & 26 & 520 & 882 & 9 \\
\hline
\end{tabular}

a) 1 Mäß $-13,9$ Liter

Quelle: Erlach-Urbare Nr. 4 und Nr. 8

Flur. Die Parzellen sind noch wesentlich größer, teilweise sogar blockförmig.

Aus der Tabelle 3 ist zu ersehen, daß auch in Gals die Betriebe als "Gut» oder «Schuppose» bezeichnet wurden. Die nähere Bezeichnung stammt aber nicht aus der Zeit der Urbaraufnahme, sondern geht vermutlich bereits ins 15 . Jahrhundert zurück.

Aus der Tabelle geht zudem die Streuung der Betriebsgrößen sowie die ganz unterschiedliche Bodenzinsbelastung hervor, wobei keine direkte Beziehung zwischen Betriebsgröße und Lehenszins festgestellt werden kann. Wie in Gampelen lassen sich auch in Gals aus der Besitzstreuung des 16. Jahrhunderts keinerlei Schlüsse ziehen zur Flurentwicklung oder sogar zur Siedlungskonzentration. Das Gewannflursystem lag bereits in seiner ausgereiften Form vor.

Von den Gütern, Schupposen und andern Landwirtschaftsbetrieben von Ins im 16. Jahrhundert haben wir nur das «Hofgut» dargestellt, obschon sich dieses um 1530 weder in der Größe noch im Grad der Zersplitterung von andern Gütern unterschied.

Die Tabelle 4 zeigt die Aufteilung des Inser Hofgutes in acht unterschiedlich große Zinseinheiten, für die sieben verschiedene Träger den Bodenzins schuldeten. Diese bewirtschafteten die Parzellen wohl auch selber. Die identischen Lehenszinse für Peter und Heini Füri lassen annehmen, daß eine größere Zinseinheit halbiert wurde, ebenso für die sehr ähnlichen Zinse für Ulrich Stachell und Willi von Gersatz bzw. Ludi Runsis und Hentzy Bruder. Die beiden Zinseinheiten von Peter Huser entstanden offensichtlich durch Teilung im Verhältnis 2: 1 .

Die Kartierung zeigt nun deutlich, daß mehrere Zinseinheiten ursprünglich Langparzellen von über $500 \mathrm{~m}$ Länge waren und daß sich diese zu einigen wenigen großflächigen Blockparzellen zusammenfü-

Tab. 4 Die Bestandteile (Bodenzinseinheiten) des «Hofgutes» Ins um 1530

\begin{tabular}{|c|c|c|c|c|c|c|c|}
\hline & \multirow[t]{3}{*}{ Zinsverantwortlicher } & \multirow{3}{*}{$\begin{array}{l}\text { Anzahl } \\
\text { Parzellen }\end{array}$} & \multicolumn{5}{|c|}{ Lehenszins } \\
\hline & & & \multicolumn{3}{|c|}{ in Mäß } & \multirow[b]{2}{*}{ Schilling } & \multirow[b]{2}{*}{ Pfennige } \\
\hline & & & Korn & Hafer & Mußkorn & & \\
\hline $\begin{array}{l}\text { vom "Hoffgutt" } \\
\text { vom "Hoffgutt" } \\
\text { vom "Hoffgutt" } \\
\text { vom "Hoffgutt" } \\
\text { vom "Hoffgutt" } \\
\text { vom "Hoffgutt" } \\
\text { vom "Hoffgutt" } \\
\text { vom "Hoffgutt" }\end{array}$ & $\begin{array}{l}\text { Ulrich Stachell } \\
\text { Peter Huser } \\
\text { Willi v. Gersatz } \\
\text { Peter Huser } \\
\text { Hentzy Bruder } \\
\text { Peter Füri } \\
\text { Ludi Runsis } \\
\text { Heini Füri }\end{array}$ & $\begin{array}{r}11 \\
26 \\
15 \\
32 \\
30 \\
7 \\
40 \\
6\end{array}$ & $\begin{array}{r}5 \\
16 \\
7 \\
32 \\
21 \\
3 \\
21 \\
3\end{array}$ & $\begin{array}{r}5 \\
16 \\
7 \\
32 \\
21 \\
3 \\
21 \\
3\end{array}$ & $\begin{array}{l}6 \\
2 \\
9\end{array}$ & 2 & $\begin{array}{r}10 \\
6\end{array}$ \\
\hline Total & & 167 & 93 & 93 & 23 & 3 & 4 \\
\hline
\end{tabular}

Quelle: Erlach-Urbar Nr. 6 
gen, die bis zu 9 ha groß waren. Die Flurnamenanalyse ergab drei «Breiten» für die Blockparzellen in den drei Zelgen und die Bezeichnung "Brühl» für die Wiesen-Blockparzelle. Nun zeigte Viktor ERNST bereits 1926 die enge Beziehung zwischen dem "örtlichen Herrengut) (Salhof, Fronhof, Meierhof, Dinghof, Zwinghof, Kehlhof) und diesen Flurnamen auf. In neuerer Zeit weisen auch ABEL und BADER diese Beziehung nach (ABEL 1967: 47; BADER 1973: 92).

Somit handelte es sich beim "Hofgut» mit größter Wahrscheinlichkeit um den Herrschaftshof von Ins, der mit dem 1185 erstmals urkundlich belegten «curia de Anesi»" identisch sein dürfte. Der Hof gehörte damals dem Kloster St. Johannsen, und sämtliche Bodenzinse waren auch im 16. Jahrhundert noch diesem Kloster schuldig.

$\mathrm{Da}$ das Kloster St. Johannsen um 1100 gegründet wurde, und auch die zweite große Grundherrschaft dieser Region erst im 11. Jahrhundert entstand ${ }^{12}$, darf angenommen werden, daß das Inser Hofgut im 11. oder spätestens im 12. Jahrhundert entstand und bereits in ein Dreizelgensystem integriert wurde. Die relativ große Siedlungsferne der Ackerparzellen im «Galgenfeld» und im «Müntschemierfeld» weisen darauf hin, daß bereits vorher eine Flur existierte. Die siedlungsnahe Lage der Parzelle "In der Breiten» könnte jedoch ein Hinweis sein, daß die dritte Zelg, das spätere «Erlachfeld», bei der Hofgutgründung noch nicht urbarisiert war. Wir vermuten deshalb für die Zeit vor dem 11. Jahrhundert lediglich eine Zweizelgenflur, wie sie sCHRöder-LembKe als Primärform in Teilen des Rheingebietes nachweisen konnte (SCHRÖDER-LEMBKE 1959: 30). Diese Hypothese wird auch durch die Flurrekonstruktion in Gampelen gestützt, wo das «Zelgli» und der «Leim» direkt am Siedlungsrand liegen, die "Gürlenzelg» als dritte Anbaueinheit jedoch abseits liegt. Die ebenfalls zur Ackerflur gehörenden «Jernet» und «Inslerenzelg» waren Bestandteile der zweiten Zelg.

Einzig in Gals fehlen Hinweise auf eine Zweizelgenflur. $\mathrm{Da}$ aber die ebenfalls vorkommenden drei «Breiten» der Siedlung direkt anschließen, vermuten wir die Urbarisierung erst mit der Anlage des Hofgutes und nehmen somit eine primäre Dreifelderwirtschaft an.

Die Zweifelderwirtschaft in Gampelen und Ins könnte auch im Zusammenhang mit dem Weinbau stehen, der in Ins für das Jahr 1009 belegt ist ${ }^{13}$ und in Gampelen aus der Siedlungslage ebenfalls als Primärnutzung vermutet wird. Der große Arbeitsaufwand des Weinbaus und das Fehlen von Düngerstoffen für die Äcker konnten dazu führen, daß pro Jahr nur die halbe Ackerflur angebaut wurde.

Zusammenfassend ergibt sich die Flurgenese für die dargestellten Siedlungen Gals, Gampelen und Ins in vier Phasen: in den vor der Jahrtausendwende entstandenen Fluren von Gampelen und Ins vermuten wir primäre Zweizelgenfluren, die im 11./12. Jahrhundert zu Dreizelgenfluren erweitert wurden. Diese vorerst noch großflächigen Blockfluren wurden durch Längsteilung in Langparzellenfluren und in einer zweiten Teilungsphase zu Kurzgewannfluren weiterentwickelt. Um 1530 waren die Gewannfluren als Hochform vorhanden. Diese Gewannflursysteme waren somit keineswegs auf Anhieb gegründete Nutzungssysteme, weder einer genossenschaftlich organisierten Siedlergruppe noch einer grundherrschaftlichen Institution. Flurzwang, gemeinsame Allmendnutzung und zahlreiche weitere Absprachen müssen vielmehr als Folge der Nutzungsintensivierung und Güterzersplitterung - beide sehr stark durch die Bevölkerungszunahme und durch das Erbrecht bedingt - gesehen werden ${ }^{14}$.

Die "Besitzrückschreibung»" ${ }^{15}$ lieferte zudem keine Hinweise auf einen hoch- oder spätmittelalterlichen Siedlungskonzentrationsprozess ${ }^{16}$. Dieser Befund wurde für das westliche Seeland auch durch die Phosphatanalyse von Bodenproben außerhalb der drei dargestellten Fluren bestätigt, die keine Hinweise auf Ortswüstungen lieferten (EGLI 1983: 156ff).

\section{SchluB}

Da einerseits die Gewannfluren vielfach nur formal als "Streifengemengeverbandsfluren" (LIENAU/UHLIG 1978: 27, im gleichen Sinn: KRENZLIN; REUSCH 1961) definiert und untersucht werden, andererseits die Zelgensysteme oft nur als Wirtschaftsform dargestellt sind (z.B. SCHRÖDER-LEMBKE 1978), ergeben sich unterschiedliche Hypothesen über Ursprung und Genese von Gewannfluren und Dreizelgensystemen. Durch die Definition des Gewannflursystems als Dreizelgensystem, das zur Gewannflur weiterentwikkelt wurde, verbanden wir Flurform und Wirtschaftsform.

Für unser Untersuchungsgebiet gelang es aufgrund der ausgezeichneten Quellenlage und der Tatsache, daß das Gebiet erst relativ spät - vermutlich erst im 7. Jahrhundert - nach einem Siedlungsunterbruch wiederbesetzt wurde (EGLI 1983: 146ff.), die Entwicklung der Mehrzelgenbrachwirtschaft und der Gewannflur aufzuzeigen. Damit konnte die Hypothese von BORN, wonach die meisten Gewannfluren sekundärer Entstehung sind (BORN 1977: 174) und die Feststellung von SCHWARZ, daß die Ausbildung des Dreizelgensystems die Entwicklung der Gewannfluren förderte (sCHWARZ 1978: 119), am lokalen Beispiel bestätigt und zudem zeitlich festgelegt werden. Es müßte nun aber noch untersucht werden, wie die Entwicklung im nordostschweizerischen und im westschweizerischen Mittelland verlief, um einerseits allfällige zeitliche Verschiebungen und andererseits Einflüsse der siedelnden Gruppen nachweisen zu können. Ob dies dort allerdings mit der gleichen Methode möglich ist, oder ob mit ganz andern Arbeitsweisen an diese Fragestellung herangetreten werden müßte, steht noch völlig offen. 


\section{Anmerkungen}

'Diese Definition entspricht der "Echten Gewannflur" von SCHARLAU (1964: 47), im Gegensatz zur "Unechten Gewannflur" mit Individualwirtschaft und Nutzungsgemenge.

${ }^{2}$ Die Unterscheidung von Dreizelgensystem und Gewannflursystem ist notwendig, weil letzteres vielerorts erst durch die sogenannte "Vergewannung", d.h. sekundär entstanden ist (KRENZLIN:, REUSCH 1961: 82), und weil das Dreizelgensystem gar keine Gewannflur bedingt (SCHRÖDER-LEMBKE 1978: 28).

${ }^{3}$ Zur Unterscheidung von Dreizelgenwirtschaft und Dreifelderwirtschaft s. BRÜHWILER 1975: 33.

${ }^{4}$ HAVERSATH bezeichnet diese Verknüpfung als "kombinierende Methode" (HAVERSATH 1984: 16).

${ }^{5}$ Die parzellenweise Urbarauswertung anhand der Katasterpläne des 18. oder 19. Jahrhunderts wurde 1961 von KRENZLIN erstmals als "Rückschreibung" bezeichnet. Die Methode ist aber wesentlich älter, so hatte zum Beispiel ZRYD bereits 1942 die Flurentwicklung von Grafenried (Kt. Bern) auf diese Art rekonstruiert.

${ }^{6}$ In den Urbaren sind die Bezeichnungen "Gut» und "Schuppose" synonym verwendet für ältere Landwirtschaftsbetriebe.

${ }^{7}$ Der Grundherr, der die landwirtschaftlichen Güter gegen einen bestimmten Bodenzins zur Bewirtschaftung verlieh, wurde im mittelalterlichen Recht als Eigentümer bezeichnet. Der Lehensnehmer, der den Landwirtschaftsbetrieb als selbständiger Unternehmer führte, war der Besitzer.

${ }^{8}$ Extraktion und Phosphatgehaltsbestimmung wurden kolorimetrisch nach dem "verbesserten Ascorbinsäureverfahren" von FREI durchgeführt (FREI 1964; ABT 1968: 20ff.).

${ }^{9}$ Diese Feststellung machte z. B. GALLUSSER (1959) auch für Fluren im Laufental.

${ }^{10}$ PFISTER (1975: 137 und Tabelle 26) berechnete für das Seeland Unterschiede der Jahreserträge im Verhältnis von 1:0,58 für die Zeit von 1755 bis 1797 . Im Mittelalter dürften diese Schwankungen noch größer gewesen sein.

"Fontes Rerum Bernensium, Bd. I, S. 474.

${ }^{12}$ Die Herrschaftsherren von Fenis, Gründer von Burg und Kloster Erlach ( - St. Johannsen) und Stammväter der Grafen von Neuenburg erhielten wahrscheinlich erst ums Jahr 1030 Lehensgüter im schweizerischen Mittelland (FLATT 1974: 99).

${ }^{13}$ Fontes Rerum Bernensium, Bd. I, S. $292 f$.

${ }^{14}$ Im gleichen Sinne z. B. auch BADER 1962: 59.

${ }^{15}$ Den Begriff prägte BALZER 1977.

${ }^{16}$ WANNER wies auch für den nördlichen Kanton Zürich nach, daß die Dörfer und "großen Siedlungen" nicht durch einen Ballungs- oder Konzentrationsprozeß im Hoch- und Spätmittelalter entstanden sind (WANNER 1984: 270f.).

\section{Quellen}

Ungedruckte Quellen im Staatsarchiv Bern:

Urbarien, Amt Erlach

Atlanten Nr. 62-72, 78, 80

Generalpläne Erlach 1, 8, 9, 24

\section{Gedruckte:}

Fontes Rerum Bernensium (1877-1956). Band I-X, Bern.

\section{Literatur}

ABEL, W. (1967): Geschichte der deutschen Landwirtschaft vom frühen Mittelalter bis zum 19. Jahrhundert. Stuttgart.

ABT, P. A. (1968): Beiträge zur Methodik der topographischen Lokalisation von Ortswüstungen. Diss. phil. Zürich.

BADER, K.S. (1962): Studien zur Rechtsgeschichte des mittelalterlichen Dorfes 2: Dorfgenossenschaft und Dorfgemeinde. Graz.

BADER, K.S. (1973): Studien zur Rechtsgeschichte des mittelalterlichen Dorfes 3: Rechtsformen und Schichten der Liegenschaftsnutzung im mittelalterlichen Dorf. Wien; Köln; Graz.

BALZER, M. (1977): Untersuchungen zur Geschichte des Grundbesitzes in der Paderborner Feldmark. (Münstersche Mittelalterliche Schriften 29). München.

BRÜHWILER, J. (1975): Der Zerfall der Dreizelgenwirtschaft im schweizerischen Mittelland. Diss. iur. Zürich.

EGLI, H.-R. (1983): Die Herrschaft Erlach. (Archiv des Hist. Vereins des Kt. Bern, 67. Bd.). Bern.

FLATT, K. H. (1974): Das Seeland im Früh- und Hochmittelalter. In: Festgabe zum Jubiläum "Das Amt Erlach 500 Jahre bernisch". Biel, 91-104.

FREI, E.; PEYER, K.; SCHÜTZ, E. (1964): Untersuchungen über die Phosphorsäurebestimmung mit Molybdänblau in Bodenextrakten. (Schweizerische landwirtschaftliche Forschung 3,3,1964).

GALLUSSER, W. (1959): Die Dreizelgenflur im Laufener Jura und ihre heutige Verbreitung im Nahbereich von Basel. In: Regio Basiliensis 1, 3-10.

HAVERSATH, J.B. (1984): Die Agrarlandschaft im römischen Deutschland der Kaiserzeit (1.-4.Jh. n. Chr.). (Passauer Schriften zur Geographie, H. 2). Passau.

KRENZLIN, A. (1961): Die Entwicklung der Gewannflur als Spiegel kulturlandschaftlicher Vorgänge. In: Berichte zur deutschen Landeskunde 27, 19-36.

KRENZLIN, A.; REUSCH, L. (1961): Die Entstehung der Gewannflur nach Untersuchungen im nördlichen Unterfranken. (Frankfurter Geographische Hefte 35). Frankfurt.

PFISTER, Chr. (1975): Agrarkonjunktur und Witterungsverlauf im westlichen Schweizer Mittelland 1755-1797. Diss. phil. Bern.

SCHARLAU, K. (1964): Die Gewannflurforschung in Hessen. In: Die Anfänge der Landgemeinde und inr Wesen Bd.I ( $=$ Vorträge und Forschungen Bd. VII), 29-51.

SCHRÖDER-LEMBKE, G. (1959): Wesen und Verbreitung der Zweifelderwirtschaft im Rheingebiet. In: Zeitschrift für Agrargeschichte und Agrarsoziologie Jg. 7, 14-31.

SCHRÖDER-LEMBKE, G. (1978): Studien zur Agrargeschichte. (Quellen und Forschungen zur Agrargeschichte, Bd. 31). Stuttgart; New York.

WANNER, K. (1984): Siedlungen, Kontinuität und Wüstungen im nördlichen Kt. Zürich (9.-15. Jh.). (Geist und Werk der Zeiten. Arbeiten aus dem Hist. Sem. der Universität Zürich, Nr. 64). Bern u. a.

ZRYD, P. (1942): Grafenried zur Zeit der Dreifelderwirtschaft. Bern. 